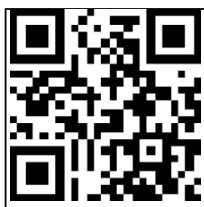

Editor's choice can to access mo free content
- Additional material is published online only. To view please visit the journal online (http://dx.doi.org/10.1136/ bjsports-2015-094927).

${ }^{1}$ Department of Epidemiology and Biostatistics, and the Ministry of Education Key Lab of Environment and Health, School of Public Health, Tongji Medical College, Huazhong University of Science and Technology, Wuhan, Hubei, China

${ }^{2}$ Department of Gastrointestinal Oncology, Hubei Cancer Hospital, Wuhan, Hubei, China

${ }^{3}$ Department of Pathology, Hubei Cancer Hospital, Wuhan, Hubei, China

\section{Correspondence to} Dr Li Liu, Department of Epidemiology and Biostatistics, and the Ministry of Education Key Lab of Environment and Health, School of Public Health, Tongji Medical College, Huazhong University of Science and Technology, No.13 of Hangkong Road, Wuhan, Hubei 430030, China gracefulliuly@163.com

TL and SW contributed equally.

Accepted 1 September 2015 Published Online First 18 September 2015

\title{
The dose-response effect of physical activity on cancer mortality: findings from 71 prospective cohort studies
}

Tingting Li, ${ }^{1}$ Shaozhong Wei, ${ }^{2}$ Yun Shi, ${ }^{1}$ Shuo Pang, ${ }^{1}$ Qin Qin, ${ }^{1}$ Jieyun Yin, Yunte Deng, ${ }^{3}$ Qiongrong Chen, ${ }^{3}$ Sheng Wei, ${ }^{1}$ Shaofa Nie, ${ }^{1}$ Li Liu ${ }^{1}$

\begin{abstract}
Background The WHO recommends moderate physical activity to combat the increasing risk of death from chronic diseases. We conducted a meta-analysis to assess the association between physical activity and cancer mortality and the WHO recommendations to reduce the latter.

Methods MEDLINE and EMBASE were searched up until May 2014 for cohort studies examining physical activity and cancer mortality in the general population and cancer survivors. Combined HRs were estimated using fixed-effect or random-effect meta-analysis of binary analysis. Associated HRs with defined increments and recommended levels of recreational physical activity were estimated by two-stage random-effects doseresponse meta-analysis.
\end{abstract}

Results A total of 71 cohort studies met the inclusion criteria and were analysed. Binary analyses determined that individuals who participated in the most physical activity had an HR of $0.83(95 \% \mathrm{Cl} 0.79$ to 0.87$)$ and $0.78(95 \% \mathrm{Cl} 0.74$ to 0.84$)$ for cancer mortality in the general population and among cancer survivors, respectively. There was an inverse non-linear doseresponse between the effects of physical activity and cancer mortality. In the general population, a minimum of $2.5 \mathrm{~h} /$ week of moderate-intensity activity led to a significant $13 \%$ reduction in cancer mortality. Cancer survivors who completed 15 metabolic equivalents of task (MET)-h/week of physical activity had a $27 \%$ lower risk of cancer mortality. A greater protective effect occurred in cancer survivors undertaking physical activity postdiagnosis versus prediagnosis, where 15 MET-h/ week decreased the risk by $35 \%$ and $21 \%$, respectively. Conclusions Our meta-analysis supports that current physical activity recommendations from WHO reduce cancer mortality in both the general population and cancer survivors. We infer that physical activity after a cancer diagnosis may result in significant protection among cancer survivors.

\section{INTRODUCTION}

Cancer is a leading disease burden in developed and developing countries with 8.2 million cancer deaths in 2012 as estimated by the WHO. ${ }^{1}$ The World Cancer Research Fund (WCRF) recently reaffirmed that the risk of cancer is affected by our lifestyles and that an active lifestyle is protective against cancer mortality. ${ }^{2}$ Specifically, an inverse association between physical activity and mortality has been discovered for breast, ${ }^{3}$ colorectal $^{4}$ and prostate cancers. ${ }^{5}$ The association was further validated by meta-analyses in breast and colorectal cancers. ${ }^{6}{ }^{7}$ However, the magnitude and intensity of physical activity most beneficial against cancer mortality is unclear. The most recent guidelines promoted by the WHO recommend a minimum of $2.5 \mathrm{~h}$ of moderate intensity physical activity (3 to $<6$ metabolic equivalents of task (MET)) or $1.25 \mathrm{~h}$ of vigorous intensity physical activity ( $\geq 6$ METs) per week or any equivalent combination for health benefits, and $5.0 \mathrm{~h}$ of moderate intensity physical activity or $2.5 \mathrm{~h}$ of vigorous intensity physical activity per week for additional health benefits..$^{8-11}$ Specific recommendations to prevent cancer mortality are still lacking. ${ }^{12}$ Accordingly, we conducted a meta-analysis of prospective studies to assess the association of physical activity with cancer mortality and to explore whether the current WHO recommendations are optimal.

\section{METHODS}

\section{Search strategy and study selection}

This meta-analysis was performed in accordance with the Preferred Reporting Items for Systematic Reviews and Meta-Analyses (PRISMA) guidelines. $^{13}$ MEDLINE and EMBASE databases were searched up to 30 May 2014 for cohort studies published in English that investigated the association between physical activity and cancer mortality. The search terms were as follows: ('exercise', 'physical activity', 'walking' or 'motor activity' with 'cancer', 'neoplasm' or 'carcinoma'). Duplicate studies were removed, and the reference lists of relevant literature and previous relevant reviews and meta-analyses were checked for additional publications of interest.

Included studies fulfilled the following criteria: (1) cohort study design, (2) physical activity (eg, leisure-time physical activity, recreational physical activity, exercise and sports, routine activity of daily living, physical activity of transportation, etc) included as a variable, (3) investigated the association between physical activity and cancer mortality (defined as deaths due to cancer) in the general population or among cancer survivors and (4) provided relative risk (RR) or HR estimates and 95\% CIs or sufficient data to calculate them. Studies were excluded if they: (1) studied a population with a chronic disease (eg, cardiovascular disease or diabetes mellitus), (2) measured physical fitness but not physical activity, (3) focused on cancer risk not cancer mortality or (4) measured only work-related physical activity. Two authors independently read the full text of all included articles to determine whether each study met the eligibility criteria outlined above. 


\section{Data extraction}

Data collection and extraction were conducted independently by two investigators, and all discordances were resolved by discussion. For each study, the following information was extracted: first author's name, publication year, cohort name, study location, study design, age at baseline, gender, number of cases or participants, number of cancer deaths, domains of physical activity, when physical activity was measured (prediagnosis (in the general population study), prediagnosis or postdiagnosis (in the cancer survival study)), amounts of physical activity at each level in different units (eg, MET-h/week, h/week, kcal/week and $\mathrm{km} / \mathrm{h}$ ), cancer type, duration of follow-up, estimate of effect (reported as a RR, HR) and the corresponding 95\% CI for the association of physical activity with cancer mortality, and adjustment variables (eg, age, body mass index (BMI) and stage). We extracted the binary estimate of the most comprehensive domain of physical activity from each study for a pooled assessment of the most active group compared with the least active group. The effect and 95\% CI were inverted for study in which the most active group was used as the reference group. Estimates from each level of recreational physical activity, the most commonly measured domain and main modifiable form of energy expenditure, were extracted for dose-response analysis. ${ }^{14}$ Lifetime results were used if a study reported the effect of physical activity at both multiple ages and over a lifetime. When a study reported separately on males and females, both risk estimates were included in the primary analysis. Additionally, in the case of multiple publications, we included the most up-to-date or comprehensive information.

\section{Assessment of risk of bias in individual studies}

We used the Newcastle-Ottawa quality assessment scale ${ }^{15}$ to assess the risk of bias in each individual study based on the following: representativeness of the exposed cohort, selection of the unexposed cohort, methods of measuring physical activity, comparability of cohorts based on design or analysis, adjustment for confounding factors (age, BMI, stage, tumour differentiation, etc), duration and adequacy of follow-up, and study end points (cancer mortality). High-quality responses earned a star with up to nine stars in total.

\section{Statistical analysis}

Binary analysis, and fixed-effect or random-effect models were used to estimate the summary HRs for associations between physical activity and cancer mortality when appropriate. ${ }^{16}$ Dose-response analyses were conducted for studies with three or more quantitative activity levels in MET-h/week and h/week, the most applicable measures of physical activity, using nonlinear random effect models. ${ }^{17}$ For each activity level, the median or mean amount of physical activity was assigned to the corresponding HR estimate. If the median or mean value was not reported, we used the midpoint of the upper and lower boundaries of each category. For studies reporting open upper boundaries for the highest category (eg, >200 min/week), we multiplied the reported lower boundary by 1.25 and used this value (eg, $250 \mathrm{~min} /$ week) as the midpoint. ${ }^{18}$ Heterogeneity in the relationship between physical activity and cancer mortality was assessed by $\mathrm{Q}$ test and quantified by $\mathrm{I}^{2}$ statistic. ${ }^{19} \mathrm{In}$ order to assess the effect of the study characteristics and quality on the reported estimates, heterogeneity was analysed by comparing the effect estimate summary from subgroup analyses. Subgroup analyses were conducted in topics that had at least three original studies and were by cancer type, gender, study location, follow-up duration ( $<10$ years, $\geq 10$ years) and when physical activity was measured (prediagnosis or postdiagnosis). To test for statistically significant potential confounders (eg, publication year, study location, follow-up duration, cancer type, when physical activity was measured, measurement of physical activity, study design and quality), meta-regression analysis ${ }^{20}$ was used to calculate ratios of risk. Publication bias was examined using Begg's test and Egger's test. ${ }^{21}$ We performed sensitivity analyses by omitting one study at a time from the initial meta-analysis. All statistical tests were two-sided and $\mathrm{p}$ values $<0.05$ were considered statistically significant. All analyses were conducted using Stata software (V.12.0; StataCorp, College Station, Texas, USA).

\section{RESULTS}

\section{Study selection}

In total, 16980 articles were initially identified in the literature search; and 10619 articles were left after removing duplicates, of which 10422 studies were not relevant to the main topic and excluded. Thirty of the remaining 197 studies were excluded due to a focus on occupational physical activity, 91 for not fulfilling the inclusion criteria and 7 for providing information from overlapping studies. Two additional articles were identified in a manual search of reference lists. Overall, 71 studies were included in the primary meta-analysis (figure 1).

\section{Characteristics of the studies}

Of the 71 prospective studies reporting on the association between physical activity and cancer mortality, 36 were general population-based studies ${ }^{\mathrm{S}[1-36]}$ and 35 studies were conducted among cancer survivors. ${ }^{S[37-71]}$ The major characteristics of these studies and reference list are shown in online supplementary table S1. In total, 3985164 participants were included in the general population-based studies and 66995 cancer deaths were observed. Nine studies were done in North

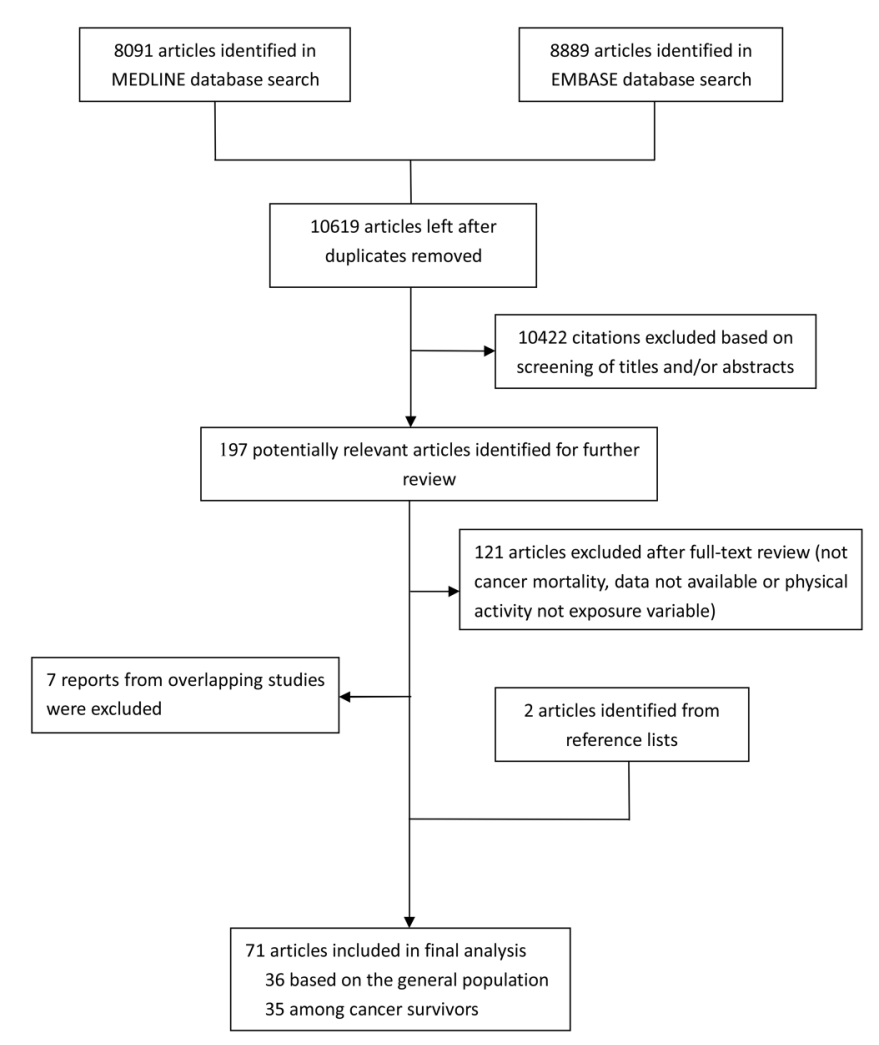

Figure 1 Flow diagram of study selection. 


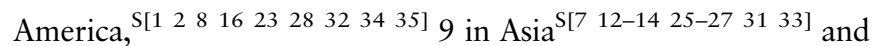

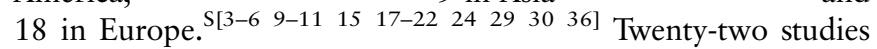
provided data on the relationship between physical activity and mortality from various cancer, ${ }^{S}\left[\begin{array}{lllllllll}1-6 & 9 & 11 & 14-16 & 19 & 21-23 & 26 & 29-31\end{array}\right.$ $\left.\begin{array}{lll}33 & 35 & 36\end{array}\right]$ with five on colorectal cancer, $\left.{ }_{1}^{12} \begin{array}{llll}24 & 26 & 27 & 34\end{array}\right]$ four on pancreatic cancer $\left.{ }^{\mathrm{S}} \begin{array}{llll}8 & 13 & 18 & 25\end{array}\right]$ and three on breast cancer. ${ }^{\mathrm{S}}\left[\begin{array}{lll}26 & 32 & 34\end{array}\right]$ In the 35 cancer survival studies, 69011 patients with cancer were included with 9516 cancer deaths. Twenty-five of these studies were conducted in North America, S[38-41 $43 \quad 44$ 48-52 54 57-65 68-71] and six in Europe. ${ }^{\mathrm{S}[45-47} 53$ 55 67] Nineteen studies were on breast cancer survival $\left.{ }^{[[37-41} 444750-55576061 \quad 67-69\right]$ and

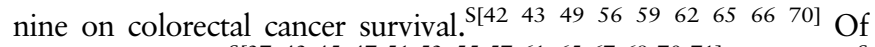
these, 24 studies, S[37-43 $45-475153-555761-6567687071] 14$ studies ${ }^{S}$

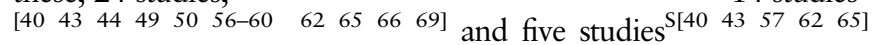
reported on the association between prediagnosis, postdiagnostic or both prediagnostic and postdiagnostic physical activity, respectively, and cancer mortality. The overall quality score ranged from 6 to 9 based on the Newcastle-Ottawa scale with 16 studies scoring 6 stars, 10 scoring 9 stars and the rest $7-8$ stars.

\section{The role of physical activity in reducing cancer mortality in the general population \\ Binary analysis}

As shown in table 1, compared to the lowest amount of physical activity, the highest amount of physical activity presented significant protection against death from cancer, with a pooled HR of 0.83 (95\% CI 0.79 to $\left.0.87, \mathrm{I}^{2}=65.6 \%\right)$. Consistent associations were confirmed by subanalyses of gender, study location, duration of follow-up and cancer type. The highest levels of physical activity reduced cancer mortality by $17 \%$ in males and females. Similarly, studies conducted in North America, Europe and Asia found a 17-19\% protective effect. Cancer mortality presented a $17 \%$ and $16 \%$ reduction in studies, respectively, with a follow-up of less than or at least 10 years. Besides, high level of physical activity reduced the mortality of colorectal cancer by $21 \%$. The heterogeneity of binary comparison was significant $\left(\mathrm{I}^{2}=65.6 \%\right)$, which, based on subgroup analysis, was mainly from North American studies. Besides study location, meta-regression did not find new sources of heterogeneity. Begg's test $(p=0.32)$ and Egger's test $(p=0.09)$ indicated no evidence for publication bias. Also, sensitivity analysis found that the pooled results did not overtly change even on omission

Table 1 Pooled measures on the relation of physical activity to cancer mortality in the general population

\begin{tabular}{|c|c|c|c|c|}
\hline & $\begin{array}{l}\text { Number of data } \\
\text { sets included }\end{array}$ & HR $(95 \%$ Cl) & $I^{2}(\%)$ & $\mathrm{p}$ Value \\
\hline Overall & 54 & 0.83 (0.79 to 0.87$)$ & 65.6 & $<0.001$ \\
\hline \multicolumn{5}{|l|}{ Sex } \\
\hline Male & 22 & 0.83 (0.75 to 0.92$)$ & 71.0 & $<0.001$ \\
\hline Female & 14 & 0.83 (0.73 to 0.94$)$ & 67.9 & $<0.001$ \\
\hline \multicolumn{5}{|l|}{ Study location } \\
\hline North America & 11 & 0.83 (0.74 to 0.93$)$ & 80.5 & $<0.001$ \\
\hline Europe & 24 & $0.82(0.75$ to 0.90$)$ & 69.3 & $<0.001$ \\
\hline Asia & 19 & 0.81 (0.76 to 0.85$)$ & 0.0 & 0.462 \\
\hline \multicolumn{5}{|c|}{ Duration of follow-up (year) } \\
\hline$<10$ & 23 & 0.83 (0.76 to 0.92$)$ & 62.6 & $<0.001$ \\
\hline$\geq 10$ & 29 & 0.84 (0.79 to 0.89$)$ & 66.2 & $<0.001$ \\
\hline \multicolumn{5}{|l|}{ Cancer types } \\
\hline Colorectal cancer & 6 & 0.79 (0.71 to 0.88$)$ & 0.00 & 0.477 \\
\hline
\end{tabular}

of the most influential study (online supplementary figures S1 and S2).

\section{Dose-response analysis}

Figure 2 shows evidence of a non-linear association between recreational physical activity and cancer mortality by MET-h/ week in general population. The HRs of cancer mortality following 5, 10, 15, 20 and 25 MET-h/week of recreational physical activity were $0.88,0.86,0.86,0.85$ and 0.84 , respectively, when compared with inactivity. The dose-response curve steeply sloped below 7.5 MET-h/week, the minimum energy expenditure of $2.5 \mathrm{~h}$ moderate physical activity per week recommended by the WHO, and then gently declined. Individuals who met the lower limit of the WHO guidelines, 7.5 MET-h/ week, had a $14 \%$ lower risk of cancer mortality. An approximate $2 \%$ reduction in cancer mortality for every $1 \mathrm{MET}-\mathrm{h} /$ week increase below 7.5 MET-h/week occurred compared to a $1 \%$ reduction in cancer mortality by every 10 over 7.5 MET-h/ week. Pooled results indicate a similar inverse relation between recreational physical activity and cancer mortality in Asians (online supplementary table S2 and figure S3). The HRs of cancer death for 5, 10, 15, 20 and 25 MET-h/week of recreational physical activity were $0.91,0.87,0.86,0.85$ and 0.84 , respectively, when compared with the lowest amount of physical activity in the Asian population. This curve shows a significant reduction below $12 \mathrm{MET}-\mathrm{h} /$ week and over $22 \mathrm{MET}-\mathrm{h} /$ week with a $1 \%$ reduction in cancer mortality for every $1 \mathrm{MET}-\mathrm{h} /$ week. A similar relationship was observed in studies within 10 years of follow-up. Other subgroups could not be analysed due to insufficient data.
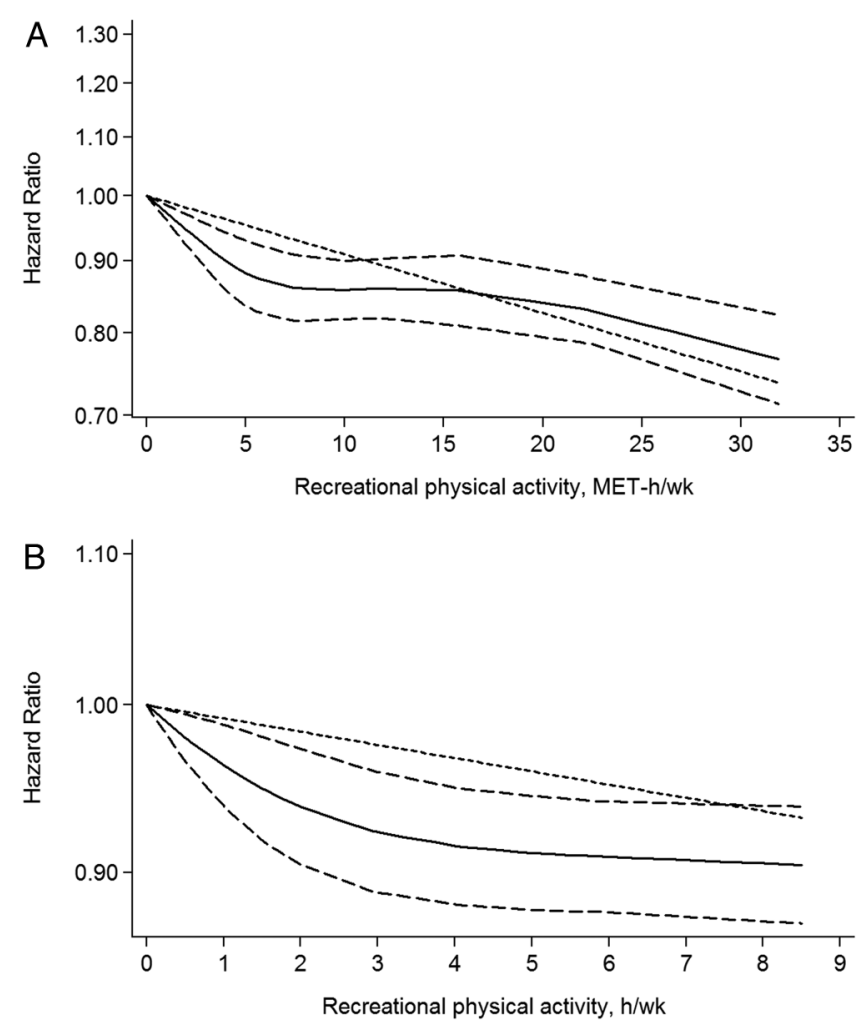

Figure 2 Dose-response relation between cancer mortality and recreational physical activity in the form of metabolic equivalents of task (MET)-h/week (A) and h/week (B) in the general population. The solid line and the long dash line represent the estimated relative risk and its $95 \% \mathrm{Cl}$. The short dash line represents the linear relationship. 
The HRs of cancer mortality for 2, 3, 4, 6 and $8 \mathrm{~h} /$ week of recreational physical activity were $0.94,0.92,0.91,0.91$ and 0.90 , respectively, compared to inactivity (online supplementary table S2). As shown in figure 2, the curve trended a decline with continuously increasing levels of recreational physical activity. Individuals who engaged in $2.5 \mathrm{~h} /$ week of recreational physical activity compared to none had a $7 \%$ lower cancer mortality. A further $2 \%$ reduction in cancer mortality was seen for every additional $6 \mathrm{~h}$ /week activity over $2.5 \mathrm{~h} /$ week. Subgroup analyses are presented in online supplementary figure S3. Cancer mortality decreased rapidly below $2 \mathrm{~h} /$ week and then declined steadily over $2 \mathrm{~h} /$ week in North Americans. A similar effect was observed in individuals following up over 10 years. Other subgroups could not be analysed due to insufficient data.

\section{The role of physical activity in reducing cancer mortality in cancer survivors \\ Binary analysis}

A strong association between high levels of physical activity and cancer mortality was observed in cancer survivors with an HR of 0.78 (95\% CI 0.72 to $0.84, \mathrm{I}^{2}=56.9 \%$ ) (table 2 ). The highest levels of physical activity reduced cancer mortality by $21 \%$ in female cancer survivors. However, we did not observe a similar association in males. The protection by physical activity against cancer death was further observed in North American studies with reduced $25 \%$ cancer mortality, but not in European. The association between physical activity and cancer mortality was not affected by duration of follow-up, which showed a $20 \%$ and $30 \%$ lower risk in participants followed up for less than and at least 10 years, respectively. A more pronounced protection from postdiagnostic physical activity $(\mathrm{HR}=0.60,95 \% \mathrm{CI} 0.50$ to $\left.0.71, \quad \mathrm{I}^{2}=53.8 \%\right)$ than prediagnostic physical activity $\left(\mathrm{HR}=0.86,95 \%\right.$ CI 0.80 to $\left.0.92, \mathrm{I}^{2}=16.7 \%\right)$ was observed among cancer survivors. This inverse association between physical activity and cancer mortality was confirmed in breast cancer survivors and colorectal cancer survivors. There was evidence of heterogeneity between cancer survival studies of highest versus lowest levels of physical activity $\left(\mathrm{I}^{2}=56.9 \%\right)$. On the basis of subgroup analyses, the studies conducted in North America are

Table 2 Pooled measures on the relation of physical activity to cancer mortality among cancer survivors

\begin{tabular}{|c|c|c|c|c|}
\hline & $\begin{array}{l}\text { Number of data } \\
\text { sets included }\end{array}$ & HR (95\% Cl) & $\mathrm{I}^{2}(\%)$ & $p$ Value \\
\hline Overall & 57 & $0.78(0.72$ to 0.84$)$ & 56.9 & $<0.001$ \\
\hline \multicolumn{5}{|l|}{ Sex } \\
\hline Female & 43 & 0.79 (0.74 to 0.84$)$ & 37.0 & 0.009 \\
\hline Male & 3 & $0.80(0.57$ to 1.12$)$ & 79.2 & 0.008 \\
\hline \multicolumn{5}{|l|}{ Study location } \\
\hline North America & 45 & 0.75 (0.68 to 0.82$)$ & 63.2 & $<0.001$ \\
\hline Europe & 8 & 0.90 (0.78 to 1.02$)$ & 0.0 & 0.679 \\
\hline \multicolumn{5}{|c|}{ Duration of follow-up (year) } \\
\hline$<10$ & 45 & 0.80 (0.74 to 0.87$)$ & 51.6 & $<0.001$ \\
\hline$\geq 10$ & 11 & 0.70 (0.55 to 0.88$)$ & 60.7 & 0.005 \\
\hline \multicolumn{5}{|l|}{ Cancer types } \\
\hline Breast cancer & 33 & $0.76(0.70$ to 0.82$)$ & 30.2 & 0.053 \\
\hline Colorectal cancer & 14 & $0.76(0.64$ to 0.90$)$ & 50.7 & 0.015 \\
\hline \multicolumn{5}{|c|}{ When physical activity was measured } \\
\hline Prediagnosis & 34 & 0.86 (0.80 to 0.92$)$ & 16.7 & 0.198 \\
\hline Postdiagnosis & 16 & $0.60(0.50$ to 0.71$)$ & 53.8 & 0.006 \\
\hline
\end{tabular}

$\mathrm{p}$ Value of Q-test for heterogeneity test. responsible for most of the observed heterogeneity. Meta-regression analysis indicated that how physical activity was measured $(p=0.01)$ was statistically significant in a multivariate model, while Egger's test suggests publication bias $(\mathrm{p}<0.001)$. Results from the sensitivity analysis did not change even if the most influential study was omitted (online supplementary figures S1 and S2).

\section{Dose-response analysis}

The pooled results show the expected inverse relationship between recreational physical activity and cancer mortality. The cancer mortality declined rapidly with a $2 \%$ reduction for every 1 MET-h/week below 10 MET-h/week followed by a plateau over $15 \mathrm{MET}-\mathrm{h} /$ week (figure 3). Compared with no recreational physical activity, 5, 10, 15, 30 and $50 \mathrm{MET}-\mathrm{h} /$ week reduced the overall cancer mortality by $18 \%, 25 \%, 27 \% 30 \%$ and $35 \%$, respectively. Subgroup analyses demonstrated that similar trends occurred in all studies included (online supplementary table S3 and figure S4). An inverse association between recreational physical activity and cancer mortality was found in females. The cancer mortality dropped rapidly with a $2 \%$ reduction for each added $1 \mathrm{MET}$-h/week below $10 \mathrm{MET}-\mathrm{h} /$ week and then stabilised at $70 \%$ for activity over $15 \mathrm{MET}-\mathrm{h} /$ week. Similarly, a protective role for recreational physical activity was observed in North Americans and cancer survivors within 10 years of follow-up. In particular, stronger protection occurred against cancer mortality with postdiagnostic physical activity compared with prediagnostic physical activity. Cancer mortality quickly decreased by $35 \%$ when individuals participated in 15 MET-h/week of recreational physical activity after diagnosis and a further 5\% reduction in cancer mortality occurred with every additional $10 \mathrm{MET}-\mathrm{h} /$ week. In comparison, the cancer mortality decreased by $21 \%$ at $15 \mathrm{MET}-\mathrm{h} /$ week of prediagnostic physical activity with no further reduction on increasing the amount of recreational physical activity (figure 4). We further explored the association between recreational physical activity and breast cancer mortality and the results were similar to the analysis of the female subgroup; the results stabilised at a $2.5 \%$ reduction in cancer mortality for every additional 1 MET-h/week below 10 MET-h/week. An inverse linear relationship was found between recreational physical activity and colorectal cancer mortality $\left(p_{\text {for non-linearity }}=0.772\right.$ ), which was statistically significant over $10 \mathrm{MET}-\mathrm{h} /$ week with a $1 \%$ reduction in cancer mortality with every additional 1 MET-h/week online

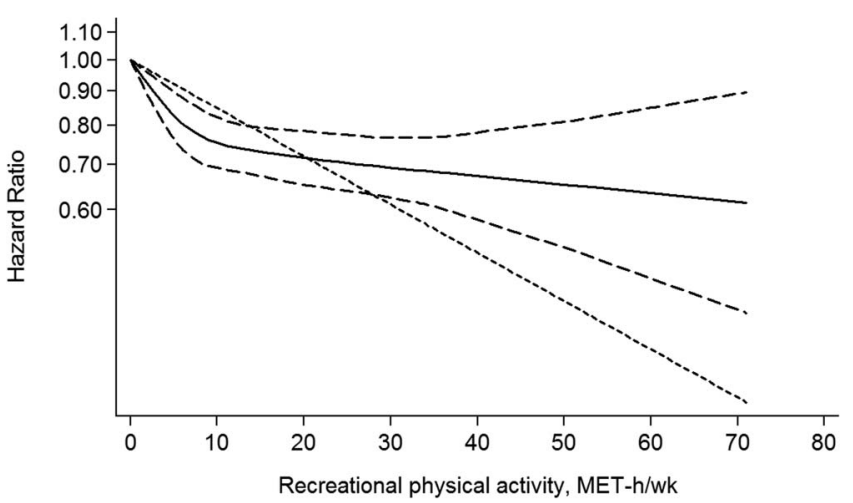

Figure 3 Dose-response relation between cancer mortality and recreational physical activity (metabolic equivalents of task (MET)-h/ week) among cancer survivors. The solid line and the long dash line represent the estimated relative risk and its $95 \% \mathrm{Cl}$. The short dash line represents the linear relationship. 

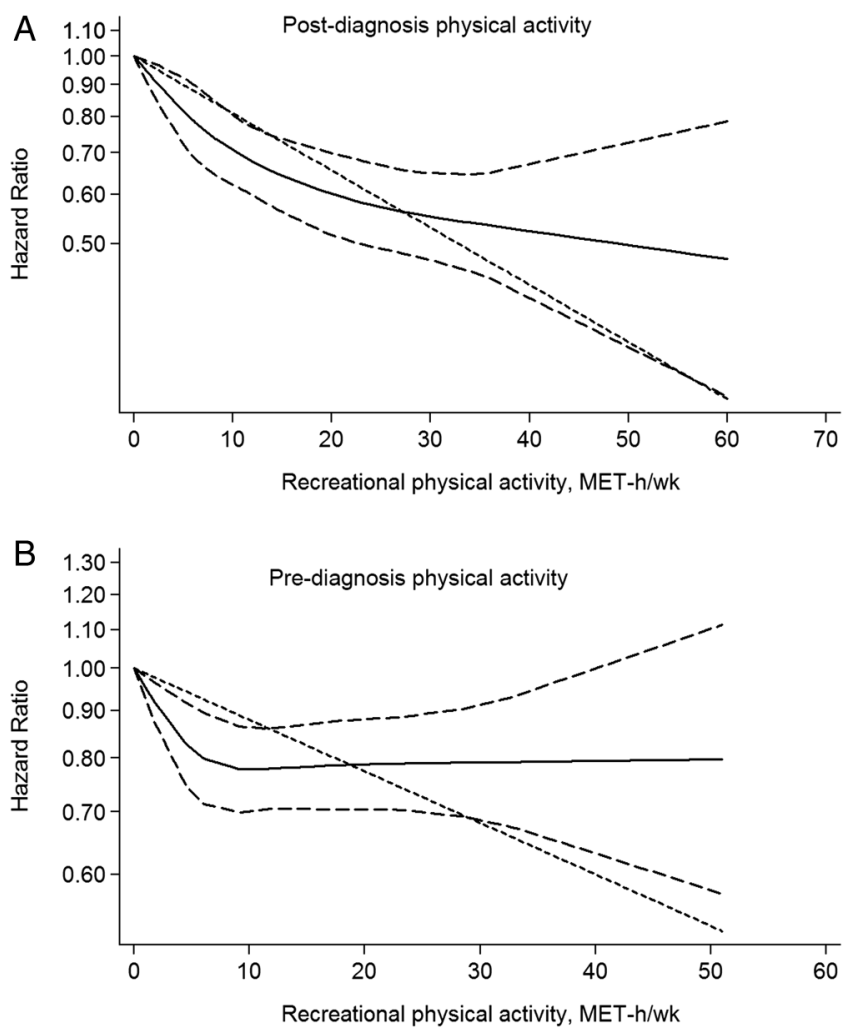

Figure 4 Dose-response relation between cancer mortality and recreational physical activity (metabolic equivalents of task (MET)-h/ week) in postdiagnosis (A) and prediagnosis (B) among cancer survivors. The solid line and the long dash line represent the estimated relative risk and its $95 \% \mathrm{Cl}$. The short dash line represents the linear relationship.

supplementary figure S4. Furthermore, we conducted subset analysis among breast cancer survivors, and a more pronounced benefit was found from postdiagnostic physical activity than prediagnostic physical activity. Compared with no recreational physical activity, 5, 10, 15 and $20 \mathrm{MET}$-h/week of prediagnostic physical activity reduced breast cancer mortality by $24 \%, 28 \%$, $29 \%$ and $30 \%$, respectively. Meanwhile, breast cancer mortality reduced by $24 \%, 32 \%, 39 \%$ and $40 \%$ when individuals participated in 5,10, 15 and $20 \mathrm{MET}$-h/week of recreational physical activity after diagnosis, respectively. Similarly, the decreasing trend in colorectal cancer mortality occurred in postdiagnostic physical activity as in overall physical activity (online supplementary figure S5).

\section{DISCUSSION}

This relatively large meta-analysis summarises the contribution of physical activity to reducing cancer mortality and quantifies the reduction in cancer mortality with incremental increases in recreational physical activity. In summary, we found that a high level of physical activity lowered the risk of cancer mortality in the general population and cancer survivors compared to inactivity. Dose-response analyses estimated the benefits for different levels of recreational physical activity by measuring MET-h/week and h/week. The results primarily showed consistent non-linear relationships between recreational physical activity and cancer mortality in the general population and among cancer survivors.

Our findings based on the general population showed that individuals undergoing the highest levels of physical activity had a $17 \%$ reduction in cancer mortality. This effect was not influenced by gender, study location or duration of follow-up. Doseresponse analyses further revealed that the cancer mortality decreased significantly by $13 \%$ and $7 \%$ in the general population that undertook 7.5 MET-h/week and $2.5 \mathrm{~h} /$ week recreational physical activity, respectively. Recent meta-analyses confirmed a similar inverse relationship between high levels of physical activity and all-cause mortality. ${ }^{18} 22$ In particular, one meta-analysis quantified the dose-response of all-cause mortality to non-vigorous physical activity and demonstrated that adhering to the WHO's recommendations contributed to a $19 \%$ reduction. $^{22}$ A recent dose-response analysis based on six studies from the National Cancer Institution Cohort Consortium found that compared with individuals reporting no leisure time physical activity, 21\% lower cancer mortality was steadily observed among those performing 1-3 times of the WHO's recommendation (7.5 to $<22.5$ MET-h/week). ${ }^{23}$ Similarly, our study, based on extensive original publications, confirmed that moderate intensity activity was associated with cancer mortality benefit in the general population as well.

The inverse relationship between physical activity and cancer mortality was also confirmed in cancer survivors. Basically, cancer survivors undertaking the highest level of physical activity had a $22 \%$ reduction in cancer mortality. In particular, evidence from the meta-analysis suggests a non-linear doseresponse of cancer mortality to recreational physical activity. Our findings confirmed and extend previous qualitative evidence, ${ }^{6} 7$ which reported a correlation between physical activity and cancer mortality in breast and colorectal cancers, respectively. In addition, our findings are consistent with recent published quantitative meta-analysis by Zhong et al, ${ }^{24}$ which revealed a similar non-linear dose-response relationship between physical activity and breast cancer mortality. The effect of recreational physical activity within a female subgroup analysis was very similar to that within the breast cancer subgroup, which suggests that they came out of similar data sources. The benefit of recreational physical activity was evident for North Americans and strongly supports the 2008 Physical Activity Guidelines for Americans, which endorses 2.5 h/week of moderate activity. ${ }^{25}$

Several mechanisms potentially explain the protection afforded by physical activity against cancer mortality. Studies cite the metabolic effects of high physical activity, including lower BMI, lower sex hormones, reduced adiposity, insulin and c-peptide levels and possibly effects on inflammation or the immune system. ${ }^{26-29}$ However, the proposed mechanisms may differ based on the type of cancer. For instance, physical activity increases insulin sensitivity. ${ }^{30}$ Higher circulating insulin and insulin-like growth factor-1 and lower insulin-binding protein level have been associated with colorectal risk in epidemiology studies. $^{31-33}$ A previous study showed higher colorectal cancer mortality among individuals with metabolic abnormalities related to insulin metabolism compared with those without hyperinsulinaemia and insulin resistance. ${ }^{34}$ In a similar way, insulin resistance may influence the risk of breast cancer recurrence and mortality, ${ }^{35}$ and physical activity is known to lower insulin levels and improve insulin sensitivity. ${ }^{36} 37$ Furthermore, exercise intervention studies have measured improvements in insulin-like growth factor 1 (IGF-I) and insulin-like growth factor binding protein 3 (IGFBP-3) and biomarkers related to cancer progression and recurrence among breast cancer survivors following high levels of exercise. ${ }^{38} 39$

Interestingly, we found that the inverse association between physical activity and cancer mortality was more pronounced 
in postdiagnostic physical activity than prediagnostic physical activity with a $26 \%$ difference. Previous meta-analyses conducted in breast and colorectal cancer survival studies clearly supported that postdiagnosis physical activity was associated with lower cancer mortality than prediagnosis physical activity. $^{7} 40$ The finding was also supported by a recently published dose-response meta-analysis in breast cancer. ${ }^{23}$ On the basis of the studies described above, there is convincing evidence that recreational physical activity after diagnosis is slightly more beneficial against cancer mortality. A possibility is that individuals who participate in physical activity after a cancer diagnosis may be motivated to change their behaviour and adopt a healthier lifestyle following a cancer diagnosis. ${ }^{41}$ Furthermore, a longitudinal study focusing on breast cancer and changes in physical activity before and after diagnosis showed that women who increased physical activity to 9 or more MET-h/week after diagnosis had lower mortality due to breast cancer even if they were inactive before diagnosis, ${ }^{42}$ and encouraged women diagnosed with breast cancer to initiate and maintain a programme of physical activity. Systematic reviews in randomised controlled trials ${ }^{43} 44$ and reviews ${ }^{45-47}$ have concluded that physical activity interventions during and after cancer therapies often result in meaningful and reliable improvements in several supportive care outcomes. These benefits include observed changes in physiological measures, objective performance indicators, self-reported functioning and symptoms, psychological well-being and overall quality of life. These findings may prompt the importance of participating in physical activity, especially after a cancer diagnosis, to gain maximum survival benefits.

\section{Strengths of the meta-analysis}

This is a large-scale meta-analysis based on 71 prospective studies. The comprehensiveness of our study is its primary strength. Besides, we provide quantified binary assessments, as well as dose-response relationships between recreational physical activity and cancer mortality. Overall, our results clarify and provide evidence for the WHO guidelines on physical activity at preventing cancer mortality for the general population and also cancer survivors. Our stratified results in the general population study further strengthen our finding by indicating consistent benefits of physical activity in different genders, study locations and durations of follow-up. In addition, we examined the difference between postdiagnostic and prediagnostic physical activity in relation to cancer mortality among cancer survivors in order to better understand the protection against cancer mortality by physical activity at different time points. Various comparisons were conducted to assess the association between physical activity and cancer mortality.

\section{Limitations of the meta-analysis}

This meta-analysis has several limitations. First, despite the inclusion of 71 studies in our meta-analysis, we were unable to assess whether the association between physical activity and cancer mortality differed by race, age or cancer type due to insufficient variation among studies in dose-response analyses. In this meta-analysis, the dose-response associations were only explored in subgroup analyses of female, North America, and breast and colorectal cancers. Second, there was significant heterogeneity for several outcomes that could not be explained by geography. The methods of how physical activity was assessed also contributed as physical activity is a complex behaviour with many components, and therefore it is difficult to accurately measure and classify the type of physical activity and its characteristics (ie, intensity, duration and frequency). Third, conclusion related to the associations between high levels of physical activity and cancer mortality in dose-response analyses should be interpreted with caution, especially in the association curve with an upward tail due to the incomplete extreme value (online supplementary figure S6), even though the the tail of the curve became flattened after omitting outliers. Furthermore, a large portion of the physical activity was self-reported; therefore, some misclassification of activity level was probable and quantitative characterisations should therefore be considered approximate in nature. Moreover, for postdiagnostic physical activity, it is possible that the sickest patients are the ones who are unable to exercise and more likely to die. However, to minimise the possibility of survival bias, the original studies conducted by Meyerhardt et $a l^{41} 48$ and Irwin et $a l^{42}$ excluded patients with cancer who either died or recurred within 1 or 2 years of physical activity assessment in their analyses, and the results were not materially altered by that procedure. Besides, all reported outcomes for postdiagnostic physical activity in this meta-analysis have been adjusted for known prognostic variables such as age and stage to reduce the influence of survival bias. Although we used adjusted estimates from included prospective studies, we cannot totally rule out potential residual confounding or confounding by unmeasured factors, such as information on treatment and more details of tumour characteristics, and those unaccounted factors may have an influence on the final results. Finally, our study suggests associations, rather than cause and effect, because of the observational nature of data.

\section{CONCLUSION}

In summary, this systematic review and meta-analysis suggests an inverse association between physical activity and cancer mortality. Quantitative data concerning the general population supports the current recommendation of physical activity equivalent to $2.5 \mathrm{~h} /$ week of moderate-intensity (3-6 MET-h/ week), which could have substantial health benefits for individuals. We also found that a minimum $2.5 \mathrm{~h} /$ week of moderate-intensity recreational physical activity conferred protection against cancer mortality among cancer survivors. Therefore, we conclude that the current recommendations concerning physical activity are generally sufficient for reducing cancer mortality. Furthermore, our study displays that physical activity performed before or after cancer diagnosis is related to reduced mortality among cancer survivors. Thus, we infer that

\section{What are the new findings?}

- By this meta-analysis based on 71 prospective studies, binary analyses determined that individuals who participated in the most physical activity had an HR of $0.83(95 \% \mathrm{Cl}$ 0.79 to 0.87$)$ and $0.78(95 \% \mathrm{Cl} 0.74$ to 0.84$)$ for cancer mortality in the general population and among cancer survivors, respectively.

- Pooled results indicate the expected inverse non-linear dose-response relationship between recreational physical activity and cancer mortality.

- Our meta-analysis supports that the current recommendation of physical activity (equivalent to $2.5 \mathrm{~h} /$ week of moderate intensity) reduces cancer mortality in both the general population and cancer survivors. We infer that physical activity after a cancer diagnosis may result in significant protection among cancer survivors. 


\section{How might it impact on clinical practice in the future?}

- Our results might be helpful to inform updates on recommendation concerning the advisable amount of physical activity to reduce cancer mortality in the general population and among cancer survivors.

- Future randomised controlled trials could be conducted to verify the role of physical activity in improving cancer mortality.

- Physical activity after diagnosis presents significant protection against cancer mortality. Therefore, physicians may consider to adopt physical active into the clinical practice of cancer treatments.

physical activity after a cancer diagnosis may result in significant protection among cancer survivors. Future randomised controlled trials are needed to verify the role of physical activity in patients with cancer. More high-quality studies are required to clarify the biological mechanisms underlying this association between physical activity and lower cancer mortality.

Contributors TL, SW (Shaozhong Wei) and LL were involved in the design of the study; TL, YS, SP, QQ, JY, YD and QC acquired data from selected studies; all authors were involved in the analysis and interpretation of the data; TL, SW (Shaozhong Wei), and LL drafted the manuscript; all authors provided critical revision of the manuscript for important intellectual content; TL, YS, SP, SW (Sheng Wei) and SN carried out the statistical analyses. All authors read and approved the manuscript. LL and TL had full access to all of the data in the study and can take responsibility for the integrity of the data and the accuracy of the data analysis. SN and $\mathrm{LL}$ are the guarantors.

Funding This work was supported by the National Natural Science Foundation of China (NSFC-81172754 to SW (Sheng Wei) and NSFC-81302491 to LL) and Independent Innovation Fund of Huazhong University of Science and Technology (2013QN001 to LL).

Competing interests None declared.

Provenance and peer review Not commissioned; externally peer reviewed.

\section{REFERENCES}

1 World Health Organization (WHO). NCD mortality and morbidity, 2012. http://www. who.int/gho/ncd/mortality_morbidity/en/ (accessed Jan 2015).

2 Wiseman M. The second World Cancer Research Fund/American Institute for Cancer Research expert report. Food, nutrition, physical activity, and the prevention of cancer: a global perspective. Proc Nutr Soc 2008;67:253-6.

3 Bradshaw PT, Ibrahim JG, Khankari N, et al. Post-diagnosis physical activity and survival after breast cancer diagnosis: the Long Island Breast Cancer Study. Breast Cancer Res Treat 2014;145:735-42.

4 Campbell PT, Patel AV, Newton CC, et al. Associations of recreational physical activity and leisure time spent sitting with colorectal cancer survival. J Clin Oncol 2013:31:876-85.

5 Kenfield SA, Stampfer MJ, Giovannucci E, et al. Physical activity and survival after prostate cancer diagnosis in the health professionals follow-up study. I Clin Oncol 2011;29:726-32.

6 Ibrahim EM, Al-Homaidh A. Physical activity and survival after breast cancer diagnosis: meta-analysis of published studies. Med Oncol 2011;28:753-65.

7 Je Y, Jeon JY, Giovannucci EL, et al. Association between physical activity and mortality in colorectal cancer: a meta-analysis of prospective cohort studies. Int $J$ Cancer 2013;133:1905-13.

8 World Health Organization (WHO). Global Strategy on Diet, Physical Activity and Health. http://www.who.int/dietphysicalactivity/factsheet_adults/en/ (accessed Jan 2015).

9 US Department of Health and Human Services. Physical Activity Guidelines Advisory Committee. Physical Activity Guidelines Advisory Committee Report. Washington DC: 2008:683. http://www.health.gov/paguidelines/ (accessed Jan 2015).

10 Ainsworth BE, Haskell WL, Whitt MC, et al. Compendium of physical activities: an update of activity codes and MET intensities. Med Sci Sports Exerc 2000;32:S498-504.

11 Haskell WL, Lee IM, Pate RR, et al. Physical activity and public health: updated recommendation for adults from the American College of Sports Medicine and the American Heart Association. Circulation 2007;116:1081-93.
12 Barbaric M, Brooks E, Moore L, et al. Effects of physical activity on cancer survival: a systematic review. Physiother Can 2010;62:25-34.

13 Moher D, Liberati A, Tetzlaff J, et al., PRISMA Group. Preferred reporting items for systematic reviews and meta-analyses: the PRISMA Statement. Open Med 2009;3: e123-30

14 Wolin KY, Yan Y, Colditz GA. Physical activity and risk of colon adenoma: a meta-analysis. Br I Cancer 2011;104:882-5.

15 Cota GF, de Sousa MR, Fereguetti TO, et al. Efficacy of anti-leishmania therapy in visceral leishmaniasis among HIV infected patients: a systematic review with indirect comparison. PLoS Negl Trop Dis 2013;7:e2195.

16 Greenland S, Robins JM. Estimation of a common effect parameter from sparse follow-up data. Biometrics 1985;41:55-68.

17 Hamling J, Lee $P$, Weitkunat $R$, et al. Facilitating meta-analyses by deriving relative effect and precision estimates for alternative comparisons from a set of estimates presented by exposure level or disease category. Stat Med 2008;27:954-70

18 Samitz G, Egger M, Zwahlen M. Domains of physical activity and all-cause mortality: systematic review and dose-response meta-analysis of cohort studies. Int $J$ Epidemiol 2011:40:1382-400.

19 Higgins JP, Thompson SG, Deeks JJ, et al. Measuring inconsistency in meta-analyses. BMJ 2003;327:557-60.

20 Sterne JA, Juni P, Schulz KF, et al. Statistical methods for assessing the influence of study characteristics on treatment effects in 'meta-epidemiological' research. Stat Med 2002:21:1513-24

21 Sterne JA, Gavaghan D, Egger M. Publication and related bias in meta-analysis: power of statistical tests and prevalence in the literature. J Clin Epidemio 2000;53:1119-29.

22 Woodcock J, Franco $\mathrm{OH}$, Orsini N, et al. Non-vigorous physical activity and all-cause mortality: systematic review and meta-analysis of cohort studies. Int I Epidemiol 2011:40:121-38

23 Arem H, Moore SC, Patel A, et al. Leisure time physical activity and mortality: a detailed pooled analysis of the dose-response relationship. JAMA Intern Med 2015;175:959-67.

24 Zhong $\mathrm{S}$, Jiang $\mathrm{T}, \mathrm{Ma} \mathrm{T}$, et al. Association between physical activity and mortality in breast cancer: a meta-analysis of cohort studies. Eur J Epidemiol 2014;29:391-404.

25 US Department of Health and Human Services. Physical activity guidelines for Americans. Washington DC: US Department of Health and Human Services, 2008.

26 Friedenreich CM, Neilson HK, Lynch BM. State of the epidemiological evidence on physical activity and cancer prevention. Eur J Cancer 2010:46:2593-604.

27 Bradley RL, Jeon JY, Liu FF, et al. Voluntary exercise improves insulin sensitivity and adipose tissue inflammation in diet-induced obese mice. Am J Physiol Endocrinol Metab 2008;295:E586-94.

28 Chu SH, Park JH, Lee MK, et al. The association between pentraxin 3 and insulin resistance in obese children at baseline and after physical activity intervention. Clin Chim Acta 2012;413:1430-7.

$29 \mathrm{Kim} \mathrm{ES}$, Im JA, Kim KC, et al. Improved insulin sensitivity and adiponectin level after exercise training in obese Korean youth. Obesity (Silver Spring) 2007;15:3023-30.

30 Helmrich SP, Ragland DR, Leung RW, et al. Physical activity and reduced occurrence of non-insulin-dependent diabetes mellitus. N Eng/ J Med 1991;325:147-52.

31 Kaaks R, Toniolo P, Akhmedkhanov A, et al. Serum C-peptide, insulin-like growth factor (IGF)-I, IGF-binding proteins, and colorectal cancer risk in women. J Natl Cancer Inst 2000;92:1592-600.

32 Ma J, Pollak MN, Giovannucci E, et al. Prospective study of colorectal cancer risk in men and plasma levels of insulin-like growth factor (IGF)-I and IGF-binding protein-3. J Natl Cancer Inst 1999:91:620-5.

33 Giovannucci E, Pollak MN, Platz EA, et al. A prospective study of plasma insulin-like growth factor-1 and binding protein-3 and risk of colorectal neoplasia in women. Cancer Epidemiol Biomarkers Prev 2000;9:345-9.

34 Trevisan M, Liu J, Muti P, et al. Markers of insulin resistance and colorecta cancer mortality. Cancer Epidemiol Biomarkers Prev 2001;10: 937-41

35 Mulligan AM, O'Malley FP, Ennis M, et al. Insulin receptor is an independent predictor of a favorable outcome in early stage breast cancer. Breast Cancer Res Treat 2007;106:39-47.

36 Boyle $\mathrm{P}$, Boniol $\mathrm{M}$, Koechlin $\mathrm{A}$, et al. Diabetes and breast cancer risk: a meta-analysis. Br J Cancer 2012;107:1608-17.

37 McTiernan A. Mechanisms linking physical activity with cancer. Nat Rev Cancer 2008:8:205-11.

38 Irwin ML, Varma K, Alvarez-Reeves M, et al. Randomized controlled trial of aerobic exercise on insulin and insulin-like growth factors in breast cancer survivors: the Yale Exercise and Survivorship study. Cancer Epidemiol Biomarkers Prev 2009;18:306-13.

39 Ligibel JA, Campbell N, Partridge A, et al. Impact of a mixed strength and endurance exercise intervention on insulin levels in breast cancer survivors. $J$ Clin Oncol 2008:26:907-12.

40 Schmid D, Leitzmann MF. Association between physical activity and mortality among breast cancer and colorectal cancer survivors: a systematic review and meta-analysis. Ann Oncol 2014;25:1293-311. 
41 Meyerhardt JA, Giovannucci EL, Holmes MD, et al. Physical activity and survival after colorectal cancer diagnosis. J Clin Oncol 2006;24:3527-34.

42 Irwin ML, McTiernan A, Manson JE, et al. Physical activity and survival in postmenopausal women with breast cancer: results from the women's health initiative. Cancer Prev Res (Phila) 2011;4:522-9.

43 Stevinson C, Lawlor DA, Fox KR. Exercise interventions for cancer patients: systematic review of controlled trials. Cancer Causes Control 2004;15:1035-56.

44 Knols R, Aaronson NK, Uebelhart D, et al. Physical exercise in cancer patients during and after medical treatment: a systematic review of randomized and controlled clinical trials. J Clin Oncol 2005;23:3830-42.
45 Galvao DA, Newton RU. Review of exercise intervention studies in cancer patients. J Clin Oncol 2005;23:899-909.

46 Schmitz KH, Courneya KS, Matthews C, et al. American College of Sports Medicine roundtable on exercise guidelines for cancer survivors. Med Sci Sports Exerc 2010;42:1409-26.

47 Wiggins MS, Simonavice EM. Cancer prevention, aerobic capacity, and physical functioning in survivors related to physical activity: a recent review. Cancer Manag Res 2010;2:157-64.

48 Meyerhardt JA, Giovannucci EL, Ogino S, et al. Physical activity and male colorectal cancer survival. Arch Intern Med 2009;169:2102-8. 